\title{
Estimación del impacto económico de los museos a través de las nuevas tecnologías de la información: el caso del museo Ene.Térmica
}

\author{
Estimating the economic impact of museums by \\ using the new information technologies: \\ The case of Ene.Térmica museum
}

\author{
Vicente COLL-SERRANO' \\ Pau RAUSELL-KÓSTER' \\ Raúl ABELEDO-SANCHIS'
}

\section{Resumen}

Cada vez es más necesario poner de relieve el potencial de la cultura como motor del desarrollo económico y su capacidad para generar ingresos y crear empleo. Por este motivo, en este trabajo se evalúa el impacto económico del museo Ene.Térmica sobre el territorio en el que se ubica, Ponferrada (Comunidad de Castilla y León, España). Para lograr este objetivo, la estimación de los distintos tipos de efectos necesarios para determinar el impacto de las actividades económicas vinculas a la existencia de Ene.Térmica se realiza, en base a la metodología de las Tablas Input-Output, mediante la herramienta Impactrimonio, una aplicación implementada sobre una plataforma web. Los resultados obtenidos indican que entre las actividades del museo y los flujos de demanda desplegados por el efecto arrastre de los visitantes se generó, entre los años 2011 y 2012, un incremento de la producción de más de 15 millones de euros, un incremento de las rentas de la población de casi 10 millones de euros y un aumento de la ocupación de 327 empleos.

Palabras clave: Impacto econômico. Impactrimonio. Museo. Tecnología de la información.

\begin{abstract}
It is increasingly necessary to emphasize the potential of culture as an engine for economic development and its ability to generate income and create jobs. For this reason, this paper evaluates the economic impact of the Ene.térmica museum in the territory in which it is located, Ponferrada (Castilla y León Community, Spain). In order to achieve this aim, the estimation of the different types of effects needed to determine the impact of economic of the activities linked to the existence of Ene.Térmica is calculated by using the tool Impactrimonio, a web-based application that applies the Input-Output Tables methodology. The results show that due to both activities of the museum and demand flows made bby the visitors, between 2011 and 2012, an increase in production was generated of over 15 million euros, an increase in the incomes of the population of nearly 10 million and an increase in employment of 327 jobs.
\end{abstract}

Keywords: Economic impact. Impactrimonio. Museum. Information technology.

\footnotetext{
1 Universidad de Valencia, Instituto de Desarrollo Local, Departamento de Economía Aplicada. Avda. Dels Tarongers s.n., 46022, Valencia, España. Correspondencia a nombre de/Correspondence to:V.COLL-SERRANO.E-mail:<vicente.coll@uv.es>.

Recebido el 25/10/2013, re-presentado el 16/7/2014y aceptado para su publicación el 30/9/2014.
} 


\section{Introducción}

Durante los últimos años los museos han sido objeto de un interés difícil de imaginar unas pocas décadas atrás. El museo moderno se muestra como una institución renovada y en constante crecimiento, perfectamente capaz de competir ya no sólo con otras alternativas culturales sino con otras formas de ocio.

Desde una perspectiva económica, un museo puede entenderse como una factoría que transforma, como cualquier otra, un conjunto de inputs a través de una serie de procesos de transformación en otro conjunto diverso de outputs. Desde el punto de vista funcional, el museo puede entenderse como una organización que, a partir de la iniciativa de promotores públicos o privados, combina capital humano, físico y financiero así como capital simbólico (el conjunto de piezas que componen una colección y el discurso que les otorga sentido). De esta manera, en un entorno tecnológico dado el museo produce un conjunto de bienes (colección, libros, audiovisuales, etc.) y servicios (de custodia, didácticos, de restauración, de investigación, etc.) con el objetivo de generar determinados impactos (estéticos, cognitivos, educativos, de entretenimiento) sobre las personas que se exponen al discurso museístico. Esto afecta también de forma indirecta tanto a aquellos que no se exponen directamente a este discurso como al territorio en el que se ubica el museo.

En los discursos de los museos se transmiten determinados valores; así, un museo de la energía, como el caso que se trata en este trabajo, pretenderá subrayar la importancia que tiene la energía en la articulación socioeconómica de un territorio. En definitiva, lo que hace es codificar y ordenar la relevancia de valores para una determinada comunidad. Pero los museos también generan impacto económico y otros efectos en el marco de la competitividad del territorio (Wavel et al., 2002; Travers, 2006; Bryan et al., 2012; Museo Guggenheim Bilbao, 2012; Tuck \& Schwartau, 2012); y hoy más que nunca, dado el actual complejo momento económico, es necesario poner en valor la cultura, puesto que hay que demostrar y estacar su potencial como motor del desarrollo económico y su capacidad para generar ingresos y crear empleo. Por este motivo, el objetivo de este trabajo es evaluar el impacto económico del museo de la energía Ene.Térmica, tanto en términos de ocupación como del valor añadido de las actividades económicas vinculadas a la existencia de dicho museo. Para ello, utilizando la aplicación Impactrimonio - una plataforma para el análisis de impactos económicos de entidades y actividades culturales diseñada y desarrollada conjuntamente por la consultora Key Productivity Konsulting (K|P|K) y el grupo de investigación en Economía de la Cultura (ECONCULT) -, se estima el impacto de Ene.Térmica-en relación al periodo 20112012.

\section{La importancia de evaluar el impacto socio-económico de los museos}

Una aproximación a los museos desde la perspectiva económica evidencia la importancia de los análisis que tratan de profundizar sobre la "racionalidad instrumental" de las instituciones que persiguen objetivos concretos, definidos en marcos lógicos diversos (acción pública, iniciativa privada o del tercer sector) mediante la utilización de determinados medios e instrumentos.

En la última década-se han sucedido actuaciones en materia de gestión de museos que apuntan a una mayor preocupación por la racionalización de sus procesos por parte de instituciones y territorios: el plan estratégico de la red de museos estatales 2004-2008 (España, 2004), el plan de calidad de los Museos Andaluces de 2003 (España, 2003), o los trabajos sobre el análisis de públicos del Laboratorio Permanente de Públicos de Museos, estudio realizado por el Instituto de Estudios Turísticos en 2007 sobre la caracterizción de los visitantes del Museo del Prado (Instituto de Estudios Turísticos de España, 2007). Las instituciones públicas muestran, tanto desde el punto de vista técnico como desde la perspectiva de los nuevos modelos de gobernanza, una creciente necesidad de fundamentar mejor sus actuaciones y de optimizar las funcionalidades de los museos en los inicios del S. XXI.

Es por ello que se propone a los responsables de la gestión de los museos emprender estrategias de medición y análisis como la que representa Impactrimonio, con el objeto de visibilizar y cuantificar el impacto económico de estas instituciones y su valor social. 
En este sentido, y pese a que la relación entre las intervenciones culturales y las dinámicas socioeconómicas es compleja y diversa, es posible sintetizar en líneas generales tres grandes ejes de interés para nuestro artículo.

En primer lugar, los museos representan un elemento de atracción de visitantes, así como de focalización de las inversiones públicas, catalizando de este modo procesos de generación de rentas y ocupación. En segundo lugar, los museos también tienen un efecto de "branding territorial", afectando en consecuencia a importantes aspectos que tienen que ver con el sentido de identidad de la población local y pertenencia a la comunidad, incidiendo sobre la cohesión social del territorio. Por último, los museos sirven de pretexto para otras intervenciones públicas, que van desde la adecuación de infraestructuras de acceso hasta la protección de elementos patrimoniales. Muchos de estos aspectos influyen notablemente en la calidad de vida de los ciudadanos.

En la actualidad, ya existe tanto una numerosa bibliografía (Frey \& Meier, 2006) como experiencia empírica sobre los resultados de los estudios de impacto económico, lo que ha permitido la adopción de una metodología común, si bien existen diferencias en función de los flujos y agentes analizados. Desde la perspectiva de Impactrimonio, se analiza el museo como una "factoría" que transforma un conjunto de recursos (inputs), a través de una serie de procesos productivos, en otro conjunto diverso de productos (outputs), de acuerdo a como está representado en la Figura 1.

El museo como unidad multiproducto multiservicio

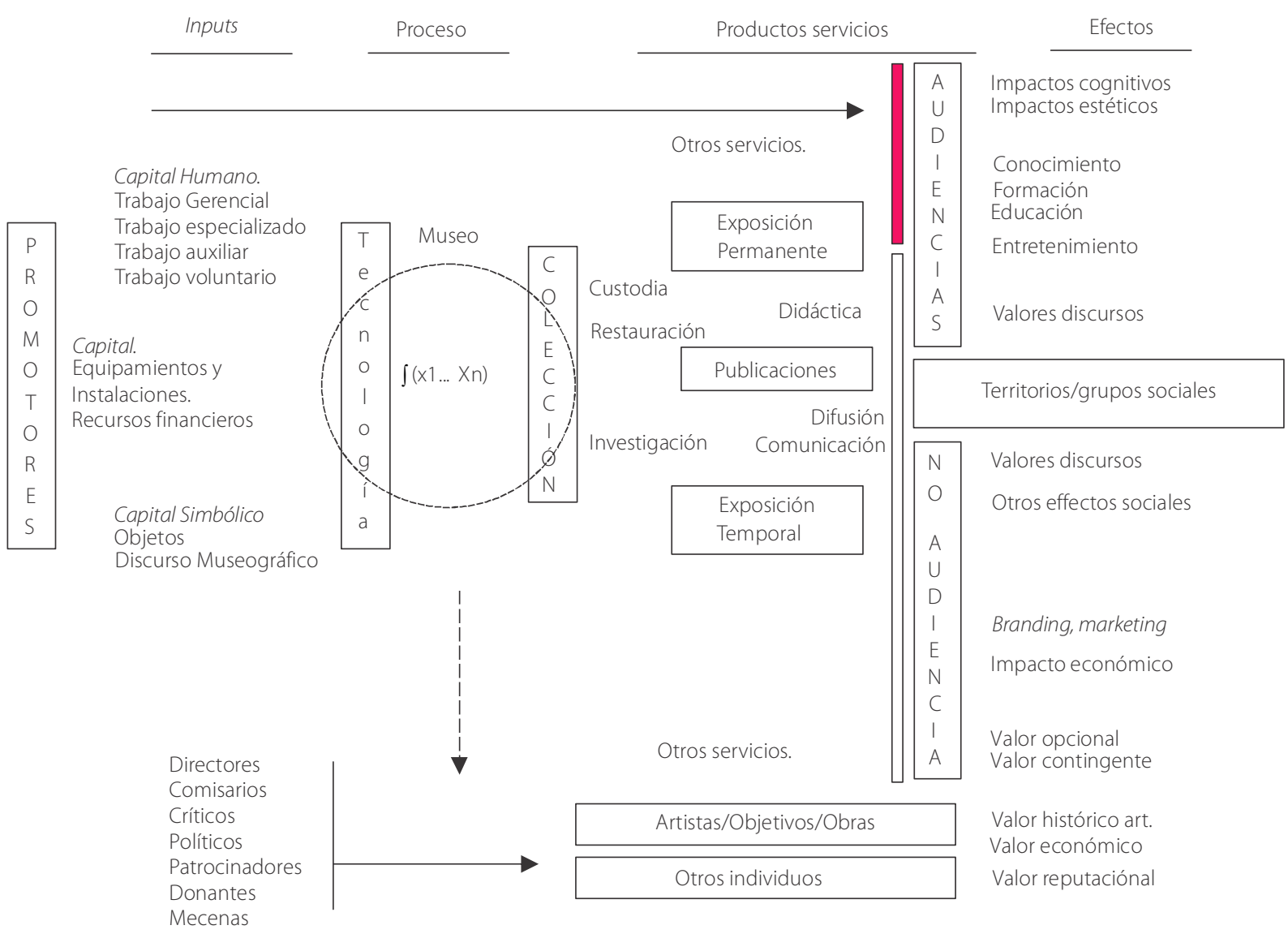

Figura 1. El museo como unidad multiproducto y multiservicio.

Fuente: Rausell-Köster et al. (2007). 
Evidentemente, deben considerarse los diversos impactos (artísticos, culturales, sociales, urbanísticos, económicos) en cualquier proceso de planificación museística, dada la íntima conexión existente entre éstos y la definición de los objetivos de la institución. Afectan, por tanto, a la gestión del "proceso productivo" del museo, de acuerdo con el esquema de la Figura 1, recursos (humanos, simbólicos, financieros), procesos tecnoproductivos (exposición, conservación, restauración, custodia, investigación), marketing y comunicación, etc.

En el actual contexto de crisis económica y replanteamiento de los modelos de financiación de la cultura, la trascendencia socio-económica del museo reclama potenciar la singularidad de su identidad como institución (de acuerdo con una estrategia de diferenciación). Al mismo tiempo deben ser impulsadas, a partir del análisis de intereses comunes y complementariedades, alianzas estratégicas de colaboración con otros museos, instituciones culturales y agentes socio-económicos, que permitan la plena puesta en valor del museo en el contexto del desarrollo local. En este sentido, las tendencias a medio plazo en los museos señalan tres grandes retos a nivel internacional (Asuaga \& Rausell-Köster, 2006):

a) Diseñar una misión y una identidad para la institución, teniendo en cuenta el valor de las estrategias de diferenciación.

b) Organizar y gestionar el museo con criterios de calidad y eficiencia, optimizando sus recursos y procesos.

c) Atraer recursos financieros, diversificando las fuentes posibles a partir de un esquema transversal (diversa naturaleza pública y privada, ámbitos de procedencia diferentes al mundo cultural) y multinivel (fuentes locales, regionales, nacionales o internacionales).

Tales retos reclaman poner en marcha una estrategia de construcción de capacidades (empowerment y capacity building) que permita afrontarlos con las máximas garantías de éxito. Es en este contexto en el que se inscribe la herramienta Impactrimonio, y cuyo funcionamiento pasa a detallarse a continuación, a partir del caso de estudio definido por el Museo de la Energía, Ene.Térmica.

\section{El museo de la energía: Ene.Térmica}

La Fundación Ciudad de la Energía (Fundación CIUDEN) es una organización, dependiente del Gobierno de España, creada en el año 2006 para ejecutar programas de $\mathrm{I}+\mathrm{D}+\mathrm{i}$ relacionados con la energía y el medio ambiente y contribuir al desarrollo económico de la comarca de El Bierzo (provincia de León, Comunidad Autónoma de Castilla y León, España). Además, entre sus propósitos está la creación y posterior gestión del Museo Nacional de la Energía, al que se ha denominado Ene.Museo.

Hasta la fecha, la realización de Ene.Museo ha dado como resultado la instalación de Ene.Térmica <http://www.enemuseo.org/index.php/es/el-museo/ espacios/enetermica>. Este proyecto museístico ha recuperado un espacio del denominado patrimonio industrial en la ciudad de Ponferrada (capital de la comarca de El Bierzo), la antigua Central Térmica de la Minero Siderúrgica de Ponferrada. Este proceso de recuperación no se ha limitado a la dimensión material, sino que conjuntamente se ha realizado un trabajo de recuperación de la memoria de todas aquellas personas que o bien trabajaron allí o bien lo hicieron en alguna de las secciones de la antigua empresa propietaria del recinto, incorporándose todo ello a los recursos museográficos.

Ene.Térmica, que se encuentra ubicada en la antigua central que estuvo en funcionamiento entre 1920 y 1971, se ha transformado en un lugar donde se cuenta la relación del carbón con la energía desde los puntos de vista tecnológico y social. Este museo debía constituir la primera fase de un proyecto museístico de mayor envergadura, el Ene.Museo Nacional de la Energía. Ene.Térmica fue abierto al público el 14 de julio de 2011. Su restauración se ha realizado respetando completamente los elementos originales de la central y ha posibilitado la recuperación, para nuevos usos culturales, de un espacio del patrimonio minero español.

Los propósitos de recuperación del patrimonio, respeto a la arquitectura y recuperación de la memoria o del patrimonio inmaterial han conducido a un reconocimiento internacional, de manera que la Comisión Europea, a través de la institución Europa 
Nostra, otorgó el premio que lleva su nombre a la Fundación Ciudad de la Energía, al considerar como singular y de especial relevancia el proyecto Ene.Térmica.

\section{Impactrimonio: una herramienta para analizar el impacto económico}

Impactrimonio es una herramienta virtual implementada en una plataforma web <www. impactrimonio.es $>$, que facilita al usuario la estimación del impacto económico derivado de una determinada actividad cultural (Rausell- Köster et al., 2013).

La metodología implementada en Impactrimonio para estimar el impacto económico se apoya en el uso de las tablas Input-Output (Pulido \& Fontela, 1993; Herrero, 2002; Seeman, 2003; Devesa, 2006; Herrero et al., 2006). Para proceder a la estimación, la herramienta Impactrimonio gestiona datos provenientes de dos fuentes distintas. En la Figura 2 se esquematiza el funcionamiento de Impactrimonio.

¿Cómo funciona?
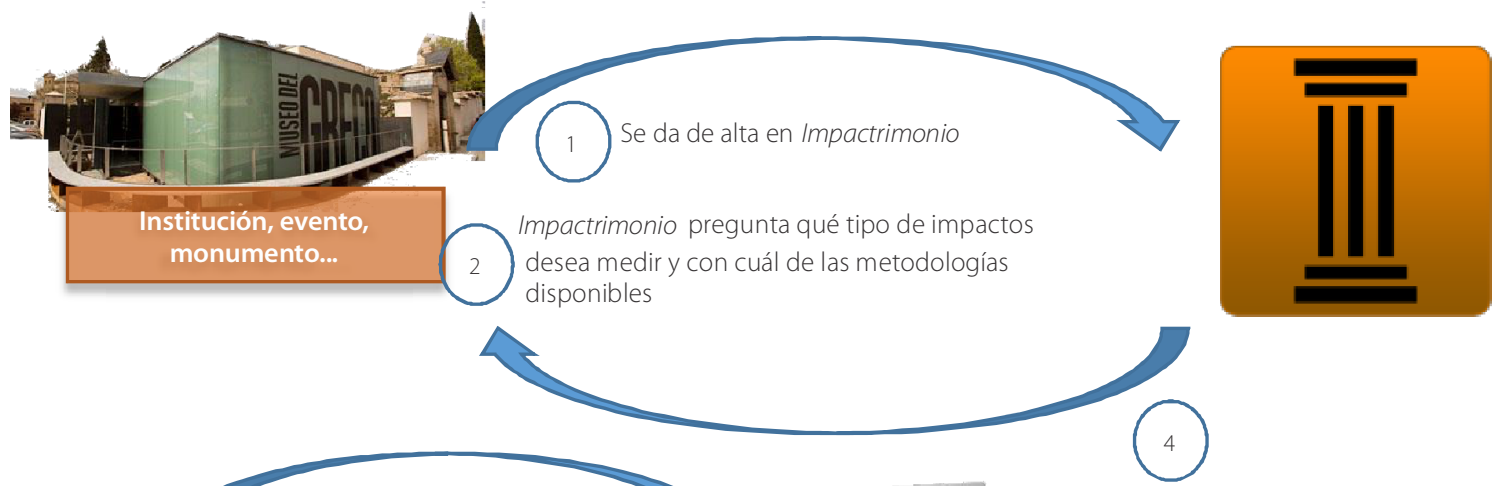

En función de la metodología y los recursos

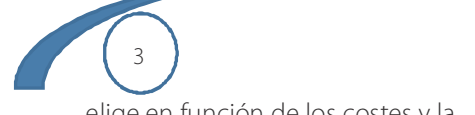

...elige en función de los costes y la información disponible

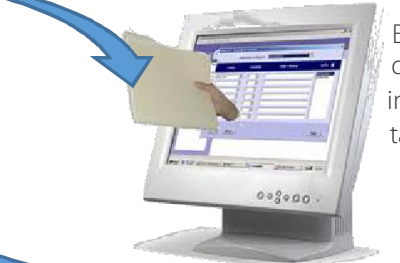
disponibles, Impactrimonio demanda información y proporciona cuestionarios y tablas de datos a completar el usuario
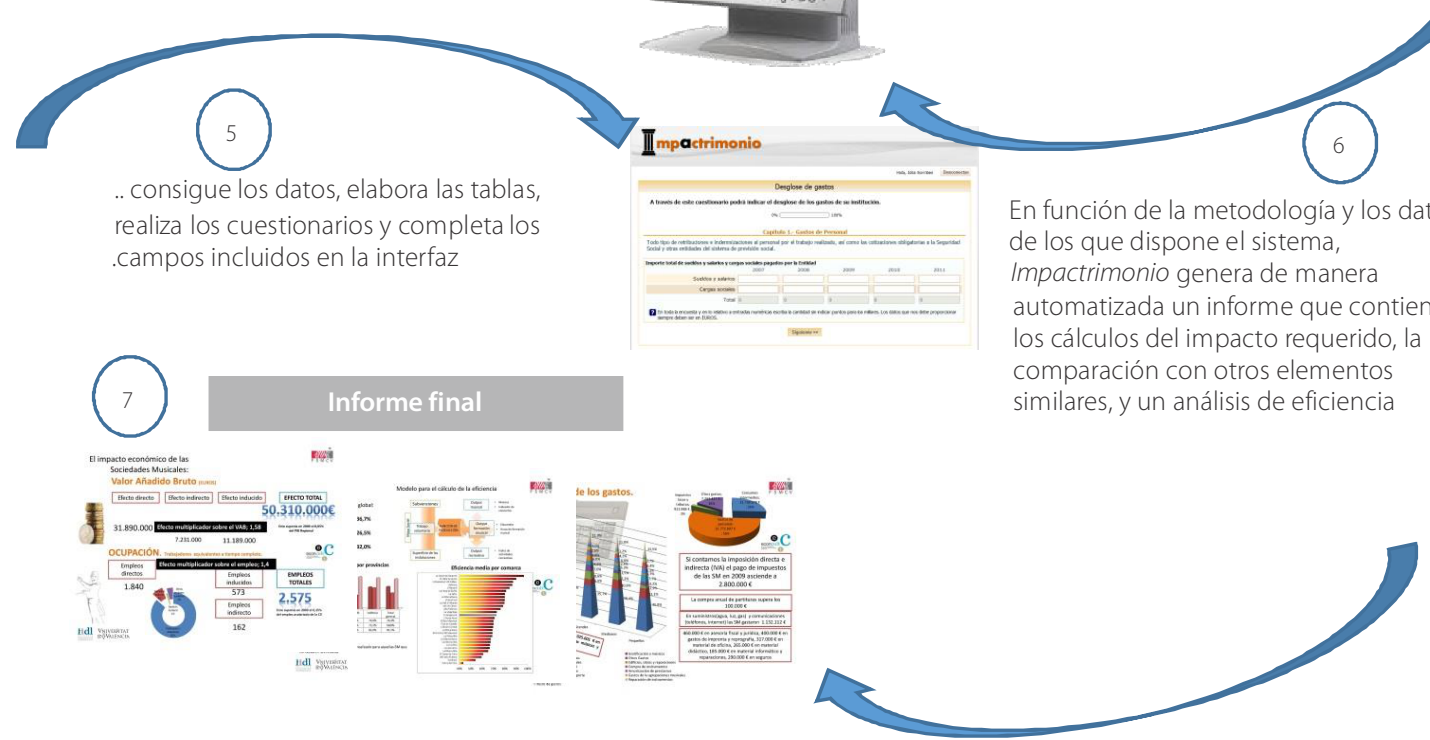

Figura 2. Funcionamiento de Impactrimonio.

Fuente: Elaboración propia (2013) 
Por un lado, Impactrimonio se alimenta de la base de datos CULTURABase del Ministerio de Cultura y de las Tablas Input-Output ( $\mathrm{TIO}$ ) de España, la cual dispone de información de más de 1.500 museos, más de 16.000 elementos patrimoniales protegidos y un número creciente de festivales. Sin embargo, para realizar el informe técnico de impacto económico es necesario acceder a los datos contenidos en la TIO de interés, en función de la localización geográfica y del ámbito de impacto de la institución a analizar. Para ello, se dispone de la tabla Input-Output a nivel nacional y de las tablas Input-Output de las distintas comunidades autónomas. Esta información ha sido obtenida del Instituto Nacional de Estadística. Dado que en origen las tablas no son homogéneas, es necesario proceder a su estandarización, para que los posteriores cálculos se realicen sobre tablas Input-Output de la misma dimensión o tamaño y sobre los mismos sectores de actividad (seleccionados atendiendo a su importancia e interés para el estudio del impacto económico de la actividad o institución cultural). A nivel técnico, para la estandarización de las tablas Input-Output se han programado diccionarios que traducen cada tabla Input-Output a los sectores de la aplicación. Estos datos se guardan en la base de datos MySQL. Asimismo, se han programado scripts que facilitan la inclusión de los datos a través de hojas de estilo.

Por otro lado, Impactrimonio gestiona los datos suministrados directamente en la aplicación por el usuario acreditado de la entidad (hasta el momento limitado a museos, festivales y elementos del patrimonio construido) para el que se solicita la valoración del impacto económico. Para capturar los datos de la institución se ha utilizado Limesurvey, una aplicación de código abierto para encuestas. Esto ha facilitado tanto la creación de las encuestas por parte del equipo de Impactrimonio como la realización de las mismas, puesto que permite acceder varias veces y completar los datos según la disponibilidad.

Los diferentes cuestionarios de Limesurvey utilizados se integran dentro de un iframe en Joomla, de forma que resulta transparente para el usuario y no tiene la sensación de que está utilizando otra herramienta. Tanto para Joomla como para Limesurvey se ha realizado un diseño similar. Ambos permiten la personalización a través de plantillas html y Cascading Style Sheets Level 2 (CSS2).
Una vez introducidos los datos solicitados al usuario, la aplicación ejecuta los cálculos matemáticos necesarios para realizar las estimaciones de acuerdo con la metodología empleada (Pulido y Fontela, 1993; Seeman, 2003). Estos datos se han programado mediante $\mathrm{R}$, un lenguaje de programación de código abierto ampliamente utilizado para cálculos estadísticos y análisis de datos. Asimismo, se ha utilizado la integración $R$ en Web rApache, un entorno para servidor web Apache que permite ejecutar scripts en lenguaje en $\mathrm{R}$ desde la web.

Impactrimonio ha contado con la participación de una veintena de instituciones colaboradoras (betatesters) a fin de comprobar (i) la pertinencia del diseño, (ii) la adecuación de la metodología, (iii) la fiabilidad de los cálculos y (iv) la usabilidad de la interfaz.

\section{La valoración del impacto económico del museo Ene.Térmica}

El esquema metodológico para el cálculo del impacto económico de Ene.Térmica en el año 2011 puede verse en la Figura 3.

Como se ha comentado en el apartado anterior, Impactrimonio utiliza, para estimar el impacto económico de Ene.Térmica, tanto la información contenida en las Tablas Input-Output - en este caso correspondiente a la Comunidad de Castilla y León por ser el territorio donde se ubica el museo - como los datos proporcionados por la propia entidad que desea evaluar su impacto económico. Estos datos son, a saber: (i) detalle de la estructura de gastos de la institución de los últimos 5 años, (ii) detalle de la estructura de ingresos de la institución de los últimos 5 años, (iii) información sobre el personal de la institución de los últimos 5 años y (iv) diversa información sobre los visitantes y la distribución del gasto que realizan.

\section{Estructura de Ingresos y gastos de Ene.Térmica}

Según la información aportada por el propio museo correspondiente al ejercicio económico del año 2011, los Ingresos Propios - esto es, los que genera el propio museo-, están integrados fundamentalmente por dos partidas: Venta de entradas y los que se ha denominado Otros ingresos procedentes de prestaciones 

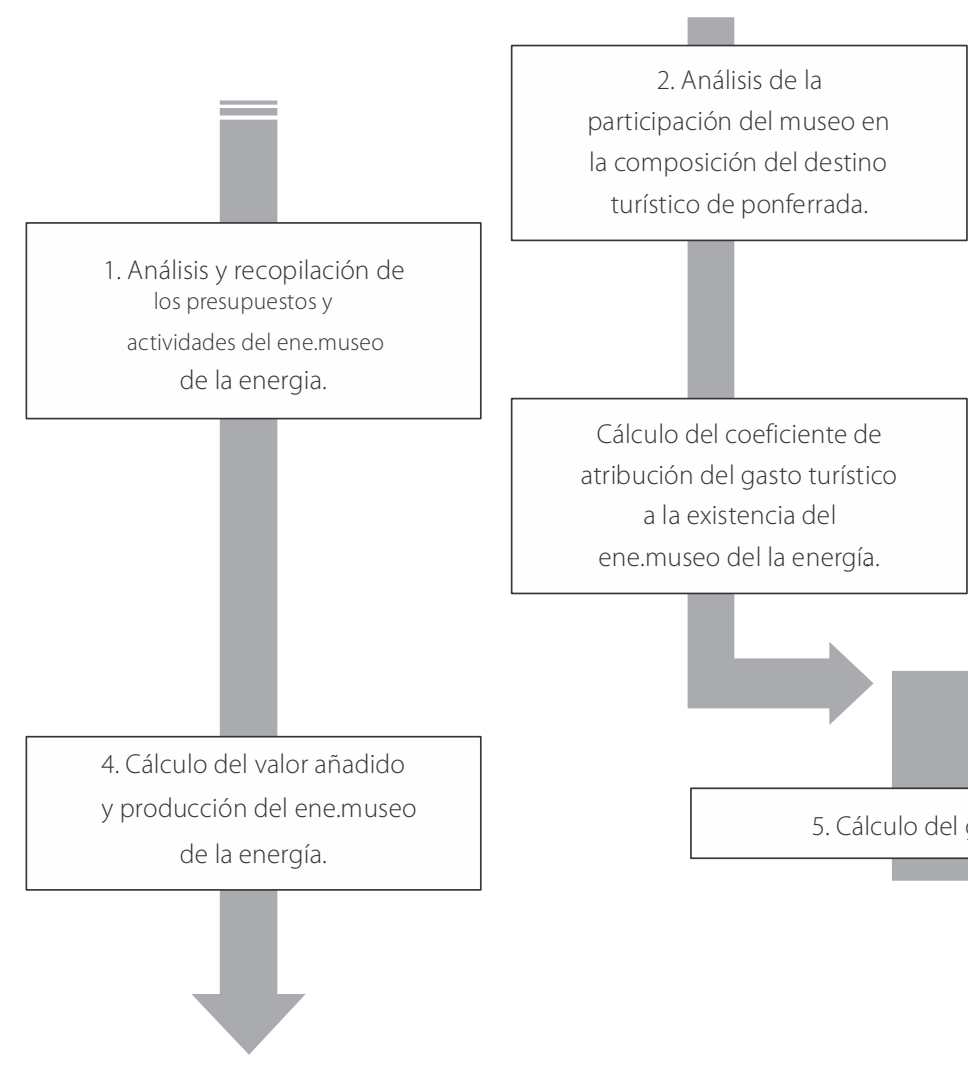

Cálculo del coeficiente de atribución del gasto turístico a la existencia del ene.museo del la energía.

3. Análisis de la estrucutra de gasto de os visitantes al ene.museo dela energía.

6. Multiplicadores tipo i y ii por sector.tablas input-outpu Castilla y León.

(Multiplicadores)

Efectos indirectos

Efectos inducidos

7. Efecto total por la existencia del Ene.Museo de la energía en término de producción, renta y empleo.

Figura 3. Metodología para el cálculo del impacto económico de Ene.Térmica.

Fuente: Elaboración propia (2013).

de servicios. El total de Ingresos propios asciende a $91.433 €$ Considerando que la estructura de los ingresos debe ser similar a la de la Fundación en la que se integra (Fundación Ciudad de la Energía), a ella es necesario remitirse para configurar la Estructura de Ingresos de Ene.Térmica, que sería como la definida en la Tabla 1. En esta tabla puede observarse que el $86,90 \%$ de los ingresos de Ene.Térmica proceden de Subvenciones, donacionesy legados de explotación; el 11,90\% serían Ingresos financieros; el 0,21\% serían los derivados de la agregación de las partidas de Ingresos por promociones, patrocinadores y colaboradores y Otros ingresos; y por último, el 0,99\% serían los Ingresos propios.
Por lo que respecta a la estructura de gastos, los egresos gastos totales por Operaciones corrientes (Gastos de personal y Gastos corrientes en bienes y servicios) ascienden a 1.454.710€ y las Dotaciones para amortización a 272.972€. En el presupuesto de gastos de 2011 cabe destacar la importante inversión realizada por el museo, que queda reflejada en el capítulo correspondiente a Inversiones reales y que suma 7.752.807€. Estas Inversiones reales principalmente se refieren a la aceptación de una serie de inmovilizado pertenecientes al patrimonio industrial de la Fundación Ciuden. La suma de estas tres partidas de gastos Operaciones corrientes, Dotaciones para amortización e Inversiones reales - asciende a 9.207.517€. 
Tabla 1. Estructura de ingresos y gastos de Ene.Térmica.

\begin{tabular}{lcc}
\hline Concepto de ingreso & Importe & $\%$ \\
\hline Ingresos propios & $91.433 €$ & 0,99 \\
Venta de entradas en 2011 & $10.783 €$ & 0,11 \\
Otros ingresos procedentes de prestaciones de servicios & $80.650 €$ & 0,88 \\
Otros ingresos & $10.027 €$ & 0,11 \\
Subvenciones, donaciones y legados explotación & $8.001 .186 €$ & 86,90 \\
Ingresos de promociones, Patrocinadores y colaboradores & $9.116 €$ & 0,10 \\
Ingresos financieros & $1.095 .753 €$ & 11,90 \\
\hline Total & $\mathbf{9 . 2 0 7 . 5 1 7 €}$ & $\mathbf{1 0 0 , 0 0}$ \\
\hline Concepto de gasto & 1 mporte & $\%$ \\
\hline Gastos de Personal & $406.212 €$ & 4,41 \\
Sueldos y salarios & $292.473 €$ & 3,18 \\
Cargas sociales & $113.739 €$ & 1,24 \\
Gastos corrientes en bienes y servicios & $775.526 €$ & 8,42 \\
Arrendamientos y cánones & $34.964 €$ & 0,38 \\
Reparaciones, mantenimiento y conservación & $153.413 €$ & 1,67 \\
Material, Suministros y otros & $583.383 €$ & 6,34 \\
Otros & $3.766 €$ & 0,04 \\
Dotaciones para amortizaciones & $272.972 €$ & 2,96 \\
Dotación amortización inmovilizado material & $257.256 €$ & 2,79 \\
Dotación amortización inmovilizado inmaterial & $15.716 €$ & 0,17 \\
Operaciones de capital (Inversiones reales) & $7.752 .807 €$ & $\mathbf{8 4 , 2 0}$ \\
\hline Total & $\mathbf{9 . 2 0 7 . 5 1 7 €}$ & $\mathbf{1 0 0 , 0 0}$ \\
\hline
\end{tabular}

Fuente: Elaboración propia a partir de Ene.Térmica (2013).

En definitiva, viendo las partidas de ingresos y gastos recogidas en la Tabla 1, puede concluirse que la fuente principal de ingresos de Ene.Térmica proviene de la transferencia de la matriz Fundación Ciuden y que el principal destino de los gastos se concentra en 2011 en las inversiones reales. Los ingresos propios resultan testimoniales y la autonomía operativa es mínima.

\section{2}

\section{Estimación del impacto económico de Ene.Térmica}

Una vez introducidos los datos relativos a ingresos, gastos, personal y visitantes en la aplicación, Impactrimonio estima los efectos directos, indirectos e inducidos para cuantificar el impacto total. Estos distintos tipos de efectos hacen referencia a los siguientes conceptos:

Efectos directos: Las inversiones y los gastos realizados por el museo o sus visitantes suponen un aumento directo de la demanda sobre las actividades que o bien son proveedoras el museo o bien son objeto directo de la demanda por parte de turistas y visitantes. En esencia, los impactos directos son consecuencia de actividades que no habrían ocurrido de no existir Ene.Térmica.

Efectos indirectos: Los sectores que reciben directamente el aumento de la demanda generan efectos indirectos sobre otros sectores, puesto que necesitan comprar más a sus proveedores para satisfacer su mayor producción. A su vez, los sectores proveedores generarán mayores demandas al resto de sectores de la economía, iniciándose de esta forma un proceso iterativo sobre el resto de la economía. La suma de los incrementos de demanda derivados de este proceso iterativo son los denominados efectos indirectos.

Efectos inducidos: Los impactos directos e indirectos a los que se ha hecho referencia anteriormente tendrán un efecto de arrastre o inducido sobre el resto de la actividad económica del territorio objeto de estudio, 
o lo que en términos técnicos se conoce como efecto multiplicador. Así, si un visitante, de fuera de la comarca de El Bierzo, utiliza un coche alquilado en esta región para desplazarse, se aloja en un hotel y disfruta de servicios diversos de restauración. Una vez visitado el museo realiza varias compras de artículos de merchandising: todos los gastos que ha realizado este visitante se computarían como efectos directos. Con dichos gastos se remuneran los factores de producción primarios (capital y trabajo), generándose renta que posteriormente se traducirá en un aumento del consumo. Este incremento del gasto en consumo volverá a producir una nueva cadena de efectos (inducidos) es lo que se denomina multiplicador de la renta. A la hora de calcular dichos multiplicadores es importante tener en cuenta el peso de las importaciones para la región, dado que cuanto menor sea el componente de productos y servicios importados mayor será el efecto multiplicador.
El impacto total asociado a un aumento de la demanda con motivo de la existencia de un equipamiento cultura, en este caso Ene.Térmica, se obtiene como la suma de los efectos directos, indirectos e inducidos. Para representar los efectos totales del impacto económico de Ene.Térmica se han separado los efectos del museo de los efectos de los turistas. Los resultados que proporciona Impactrimonio de las estimaciones de los distintos tipos de efectos han sido sintetizados tal y como se muestran en la Figura 4.

Es obvio que el principal efecto económico se debe a los gastos directos de la institución museística. El efecto de los visitantes es bastante limitado. Hay que tener en cuenta que casi el 50\% de los visitantes del museo son locales; y que, en los supuestos de imputación (a partir del cuestionario realizado a los usuarios), se ha estimado que aproximadamente sólo el 30\% del gasto turístico es atribuible a la existencia de Ene.Térmica. De

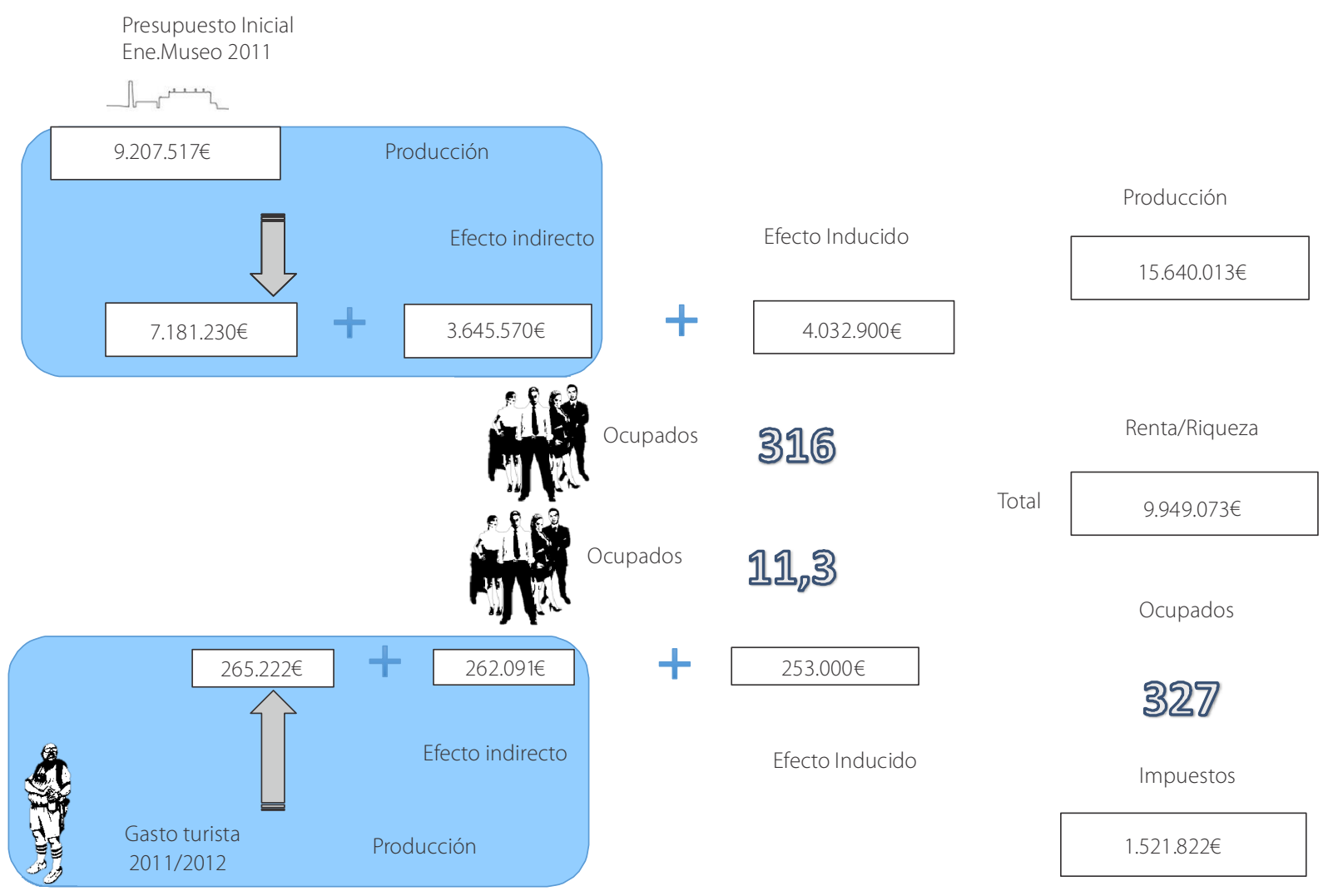

Figura 4. Estimación del impacto económico de Ene.Térmica. Fuente: Elaboración propia (2013). 
los visitantes de fuera de la Comunidad de Castilla y León, se ha supuesto que la mitad de los gastos de transporte los realizan en la comunidad de origen.

Como puede verse en la Figura 4, la actividad del museo Ene.Térmica se estima supone un incremento de la producción de 14.859.700€; de los cuales 7.181.230€ corresponderían a los efectos directos, 3.645.570€ a los efectos indirectos y los restantes 4.032.900€ a los efectos inducidos. Si a esto se añade el efecto que sobre la producción ejercen los visitantes al museo, resulta que el incremento de producción derivada de la existencia de Ene.Térmica se eleva hasta alcanzar los 15.640.013€. Es decir, calculando un multiplicador sobre la producción que ponga en relación el efecto total con el presupuesto inicial de Ene.Térmica, el valor de este multiplicador sería de 1,7. Este valor indica que por cada euro de presupuesto del museo se consiguen movilizar recursos por valor de 1,7 euros.

Por otro lado, en términos de riqueza, el efecto total conjunto de Ene.Térmica y turistas se estima en 9.949.073€. En este caso, el impacto de Ene.Térmica en generación de riqueza es menos significativo, puesto que el valor del multiplicador sobre la renta es de 1,08; esto es, por cada euro puesto en circulación por el museo se genera una riqueza sobre el territorio de 1,08 euros.

Por último, se ha estimado que la actividad de Ene.Térmica crearía 316 puestos de trabajo y que la actividad turística derivada generaría en torno a 11 empleos, lo que daría un número total de ocupados de 327 personas. Además, debe destacarse que hay un impacto sobre la capacidad impositiva, llegando a generar algo más de 1,5 millones de euros de recaudación, de los que aproximadamente el 75\% correspondería a imposición sobre rentas y el restante $25 \%$ vendría derivado de la imposición sobre la producción y los productos.

\section{Conclusión}

Ene.Térmica, que se encuentra en funcionamiento desde Julio de 2011, debía constituir una primera fase de una propuesta museística de mayor envergadura, el Ene.Museo Nacional de la Energía, truncada a la fecha de hoy. Con todo, Ene.Térmica tiene un adecuado engarce con el territorio en el que se ubica. La recuperación patrimonial material y de la memoria de la central térmica establecen un vínculo con la tradición minera secular de la comarca, pero al mismo tiempo conectan con un conjunto de vivencias y experiencias relativamente recientes de la población local de manera tal que a partir de la rehabilitación patrimonial logra establecer vínculos experienciales y vivenciales de alta intensidad.

En cuanto a la evaluación del impacto económico de Ene.Térmica sobre el territorio, el impacto de mayor envergadura no se deriva, como era previsible, de los efectos de arrastre de los turistas sino de la propia actividad del museo. Lo que sí resultan son cifras nada despreciables en un contexto de extrema contracción económica como se está viviendo en España en este periodo. Entre las actividades del museo y los flujos de demanda desplegados por el efecto arrastre de los visitantes se generó entre los años 2011 y 2012 un incremento de la producción de más de 15 millones de euros, una suba de las rentas de la población de casi 10 millones de euros y un aumento de la ocupación de 327 empleos equivalentes a tiempo completo.

La estimación del impacto económico del museo Ene.Térmica se ha realizado utilizando Impactrimonio, una herramienta virtual implementada en una plataforma web que estima los distintos tipos de efectos a considerar para determinar el impacto económico apoyándose en el uso de las tablas Input-Output. El modelo Impactrimonio es totalmente reproducible y adaptable a otros contextos territoriales. Para ello, el requisito necesario es disponer de las correspondientes tablas Input-Output y de los datos económicos de la entidad para la que se desea evaluar el impacto económico. Actualmente, ha concluido la fase de prototipo de Impactrimonio y se está trabajando en la programación que permita la generación automática de los informes de impacto.

\section{Agradecimientos}

Esta investigación ha recibido financiación de la Subdirección General de Patrimonio Artístico del Ministerio de Educación, Cultura y Deporte y del Ministerio de Economía y Competitividad (Plan Nacional de I+D+i 2008-2011, proyecto CSO2012-39373-C04-3). Los autores desean agradecer a Francisco Marco-Serrano, profesor y gerente de la consultora Key Productivity Konsulting, su participación en el diseño y desarrollo de Impactrimonio. 
Asuaga, C.; Rausell-Köster, P. Un Análisis de la gestión de las instituciones culturales: El caso específico de los museos. Revista Iberoamericana de Contabilidad de Gestión, n.8, p.83-104, 2006. Disponible en: <http://www.observatorio-ibero americano.org/RICG/No_8/Carolina\%20 Asuaga.pdf>. Acceso en: 15 jul. 2014.

Bryan, J.; Munday, M.; Bevins, R. Developing a framework for assessing the socioeconomic impacts of museums: The regional value of the flexible museum. Urban Studies, v.49, n.1, p.133-151, 2012. Available from: <http://usj.sagepub.com/ content/49/1/133.full.pdf+html>. Cited: Jul. 15, 2014.

Devesa, M. El impacto económico de los festivales culturales. Madrid: Datautor, 2006.

España. Junta de Andalucía. Plan de calidad de los Museos Andaluces. Sevilla: Consejería de Educación, Cultura y Deporte, 2003. Disponible en: <http://www.juntadeandalucia.es/ export/drupaljda/6029_0205_plan_de_calidad_2-1.pdf>. Acceso en: 21 abr. 2015.

España. Ministerio de Cultura. Plan estratégico de la red de museos estatales 2004-2008. Madrid: Ministerio de Cultura, 2004. Disponible en: <http://www.mcu.es/museos/docs/ PlanEstrategico Museos.pdf>. Acceso en: 15 jul. 2014.

Frey, B.; Meier, S. The Economics of Museums. In: Ginsburgh, V.A.; Throsby, D. (Ed.). Handbook of the Economics of Art and Culture. Amsterdam: Elsevier, 2006. v.1 p.1018-1047.

Herrero, L.C. La economía de la cultura en España: una disciplina incipiente. Revista Asturiana de Economía, v.23, p.147-175, 2002. Disponible en: <http://www.revistaasturianade economia.org /raepdf/23/P147-176.pdf>. Acceso en: 15 jul. 2014.

Herrero, L.C. et al. The economic impact of cultural events: A case-study of Salamanca 2002: European capital of culture. European Urban and Regional Studies, v.13, n.1, p.41-57, 2006. Available from: <http://eur.sagepub.com/content/13/1/ 41.full.pdf+html>. Cited: Jul. 15, 2014.

Instituto de Estudios Turísticos de España. Estudio sobre los visitantes del Museo del Prado del IET 2007. Madrid: Instituto de Estudios Turísticos de España, 2007. Disponible en: <http:// www.iet.tourspain.es/es-es/estadisticas/otrasestadisticas/ museodelprado/paginas/default.as px>. Acceso en: 21 abr. 2015.

Museo Guggenheim Bilbao. Impacto de las actividades del museo Guggenheim Bilbao en la economía del País Vasco, 2012. Disponible en: <http://prensa.guggenheim-bilbao.es/src/ uploads/2012/12/Impac_econ_ESP_2010.pdf>. Acceso en: 15 jul. 2014.

Pulido, A.; Fontela, E. Análisis input-output: modelos, datos y aplicaciones. Madrid: Pirámide, 1993.

Rausell-Köster, P. et al. Cultura: estrategia para el desarrollo local. Madrid: Agencia Española de Cooperación Internacional, 2007. Disponible en: <http://www.cervantesvirtual.com/obra/ cultura-estrategia-para-el-desarrollo-local-0/>. Acceso en: 21 abr. 2015.

Rausell-Köster, P. et al. Impactrimonio: las nuevas tecnologías de la información aplicadas a la valoración del impacto económico de la cultura. El Profesional de la Información, v.22, n.4. p.309-314, 2013. Disponible en:<http://www.elprofesional delainformacion.com/contenidos/2013/julio/index.htmls. Acceso en: 21 abr. 2015.

Seeman, B. Economic impact of arts. In: R. Towse (Ed.). A handbook of cultural economics. Cheltenham: Edward Elgar, 2003. p.224-231.

Travers, T. Museums and galleries in Britain: Economic, social and creative impacts. London: London School of Economics, 2006. Available from: <http://www.nationalmuseums.org.uk/ media/documents/publications/museums_galleries_in_ britain_travers_2006.pdf>. Cited: Jul.15, 2014.

Tuck, B.: Schwartau, B. Economic contribution of museums in Minnesota: A report of the economic impact analysis program. University of Minnesota, 2012. Available from: <http:// www.tourism.umn.edu/prod/groups/cfans/@pub/@cfans/ @tourism/documents/asset/cfans_asset_386487.pdf>.Cited: Jul. 15, 2014.

Wavel, C. et al. Impact evaluation of museums, archives and libraries: Available evidence project. Aberdeen: The Robert Gordon University, 2002. Available from: <http://www4. rgu.ac.uk/files/imreport.pdf>. Cited: Jul. 15, 2014. 

